BRIEF REPORTS

\title{
A SUBCUTANEOUS NODULE WITH WHIPPLE'S DISEASE: KEY TO EARLY DIAGNOSIS?
}

\author{
ARMIN E. GOOD, THEODORE F. BEALS, JERRY L. SIMMONS, and MOSTAFA A. H. IBRAHIM
}

Arthritis usually precedes the other manifestations of Whipple's disease (WD). During this "lead time," which may extend as long as 35 years (1), the correct diagnosis may be virtually impossible without recourse to synovial biopsy. There are scattered accounts of subcutaneous nodules with Whipple's disease, but only a single published report of a nodule biopsy-a brief notation that "the changes were consistent with rheumatoid arthritis" (2).

This is a report of a patient with a nodule that followed the onset of oligoarthritis by 3 years and preceded the appearance of diarrhea and weight loss by 12 years. Biopsy of the nodule utilizing electron microscopy disclosed specific diagnostic features of Whipple's disease.

\section{CASE REPORT}

The patient, a man born in 1923, was free from rheumatic symptoms until 1963, when he began to have attacks of pain and swelling in both knees lasting up to 3 days. By 1968 these attacks also involved the wrists, small hand joints, ankles, and feet, and lasted up to 5 days. The pain and swelling tended to migrate from joint to joint. Usually only one or two joints were involved at any given time. By 1978 the attacks in the wrists became especially frequent and more prolonged; strenuous manual work appeared to precipitate these symptoms.

In 1966 a painless soft nodule appeared over the right tibial tubercle and enlarged slowly during the ensuing 12 years.

From the Departments of Medicine and Pathology, Veterans Administration Medical Center, and the University of Michigan Medical School, Ann Arbor, Michigan.

Armin E. Good, MD; Theodore F. Beals, MD; Jerry L. Simmons, MD; Mostafa A. H. Ibrahim, MD.

Address reprint requests to Dr. Armin E. Good, Rheumatology Section, Veterans Administration Medical Center, 2215 Fuller Rd., Ann Arbor, Michigan 48105.

Submitted for publication November 7, 1979; accepted in revised form March 13, 1980.
In 1978 the patient noted weight loss. Four months before admission in December 1978, diarrhea appeared with foamy, light colored, foul-smelling, liquid stools.

On admission the patient appeared mildly chronically ill. A $2 \times 4 \mathrm{~cm}$ soft rubbery nodule was situated between the right tibial tubercle and the overlying skin (Figures 1A and B). The wrists were minimally swollen and showed motion pain and limited range of motion. There was no lymphadenopathy or abnormal cutaneous pigmentation.

The erythrocyte sedimentation rate, serum B12, folate, and rheumatoid factor were normal or negative. Low values were reported for the hematocrit, serum calcium, phosphorus, cholesterol, and carotene. The prothrombin time was prolonged, and the D-xylose absorption test was abnormal. X-rays of the spine, knees, and ankles showed slight degenerative changes. The sacroiliacs were normal. $\mathrm{X}$-rays of the hands and wrists showed nonspecific cystic changes.

A technetium pertechnetate scan showed increased uptake at the wrists, right ankle, a metacarpophalangeal, and a distal interphalangeal joint, and the subcutaneous nodule in the right pretibial region.

The diagnosis of Whipple's disease was established by small bowel biopsy showing foamy histiocytes with periodic acid Schiff (PAS) positive material; bacilli and characteristic phagosomes were seen by electron microscopy.

The patient responded promptly to penicillin, 1.2 million units given intramuscularly per day, with resolution of diarrhea, progressive weight gain, clearing of arthralgia, and improved range of wrist motion.

The nodule over the right tibial tubercle was biopsied and submitted for histologic and ultrastructural analysis in Millonig's phosphate/sucrose formalin (3). One millimeter cubes were further fixed in glutaraldehyde and osmium tetroxide and embedded in plastic for electron microscopy.

Sections of paraffin-embedded tissue were 


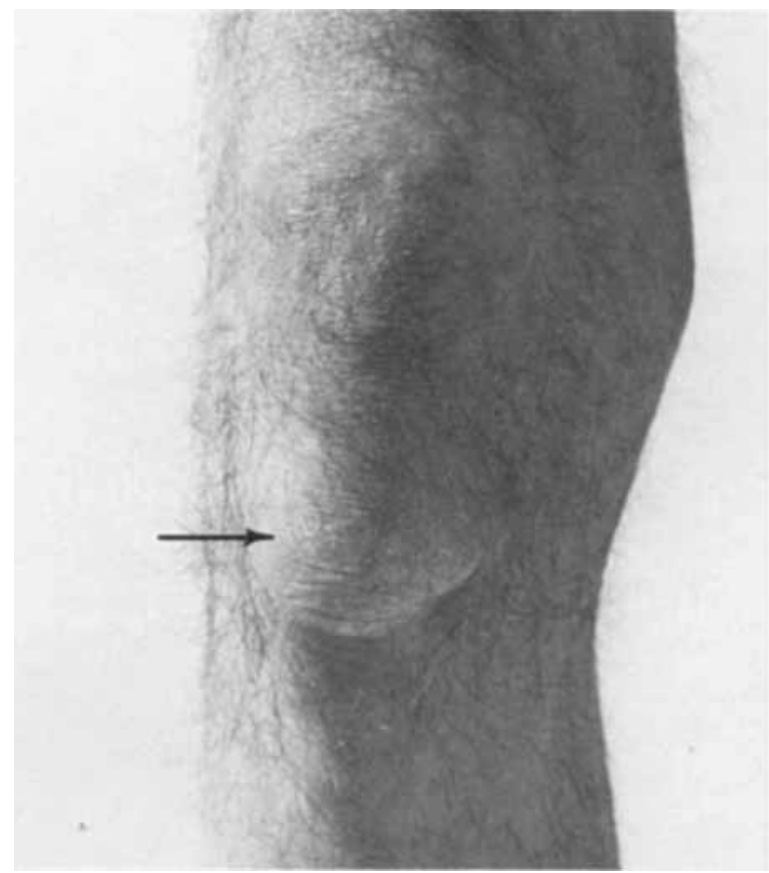

$\mathbf{A}$

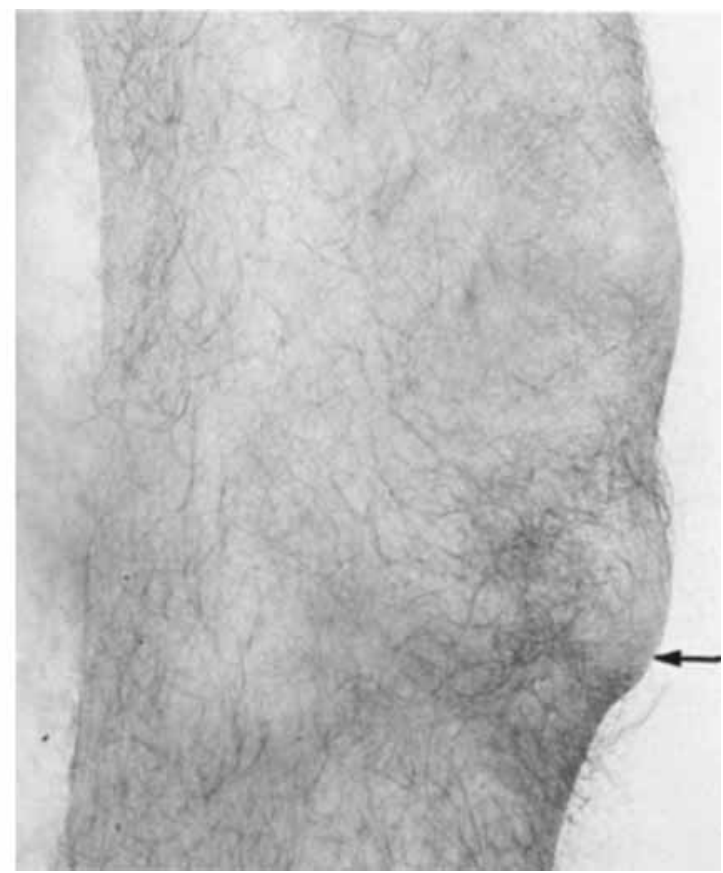

B

Figure 1. Right knee, frontal (A) and lateral (B) views, showing nodule (arrowed) overlying the tibial tubercle.

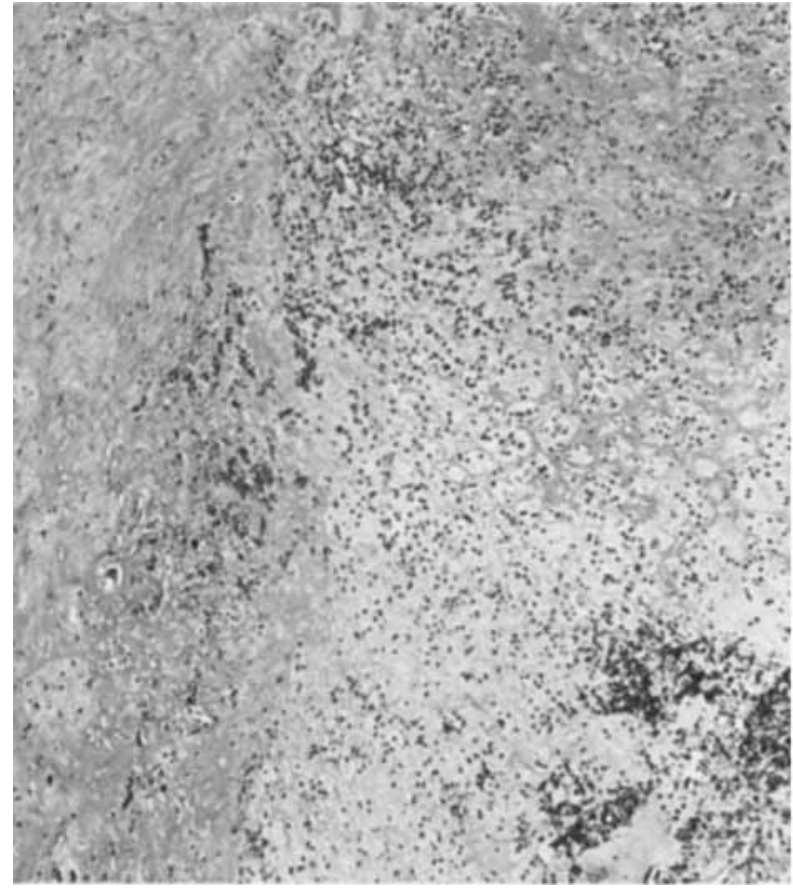

$\mathbf{A}$

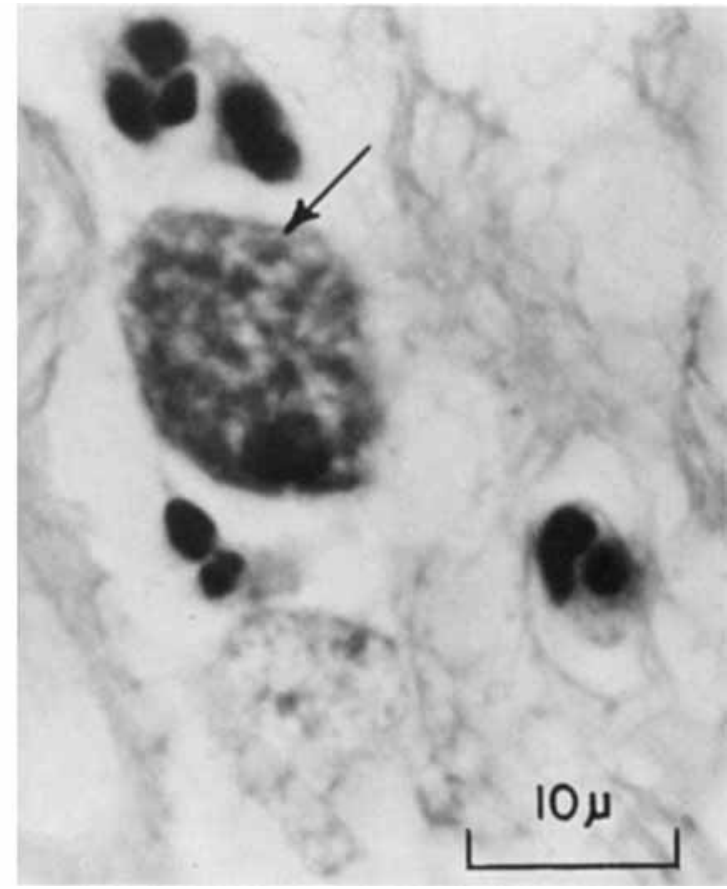

B

Figure 2. Histopathology of nodule. A, Low power view of connective tissue capsule (left) and focal aggregates of polymorphonuclear leukocytes (upper right) and pockets of foamy macrophages (lower right) $(\times 200)$. B, Foamy macrophages filled with PAS positive, diastase resistant, intracytoplasmic curvilinear bodies (arrow) consistent with Whipple's disease. 
$\mathbf{A}$
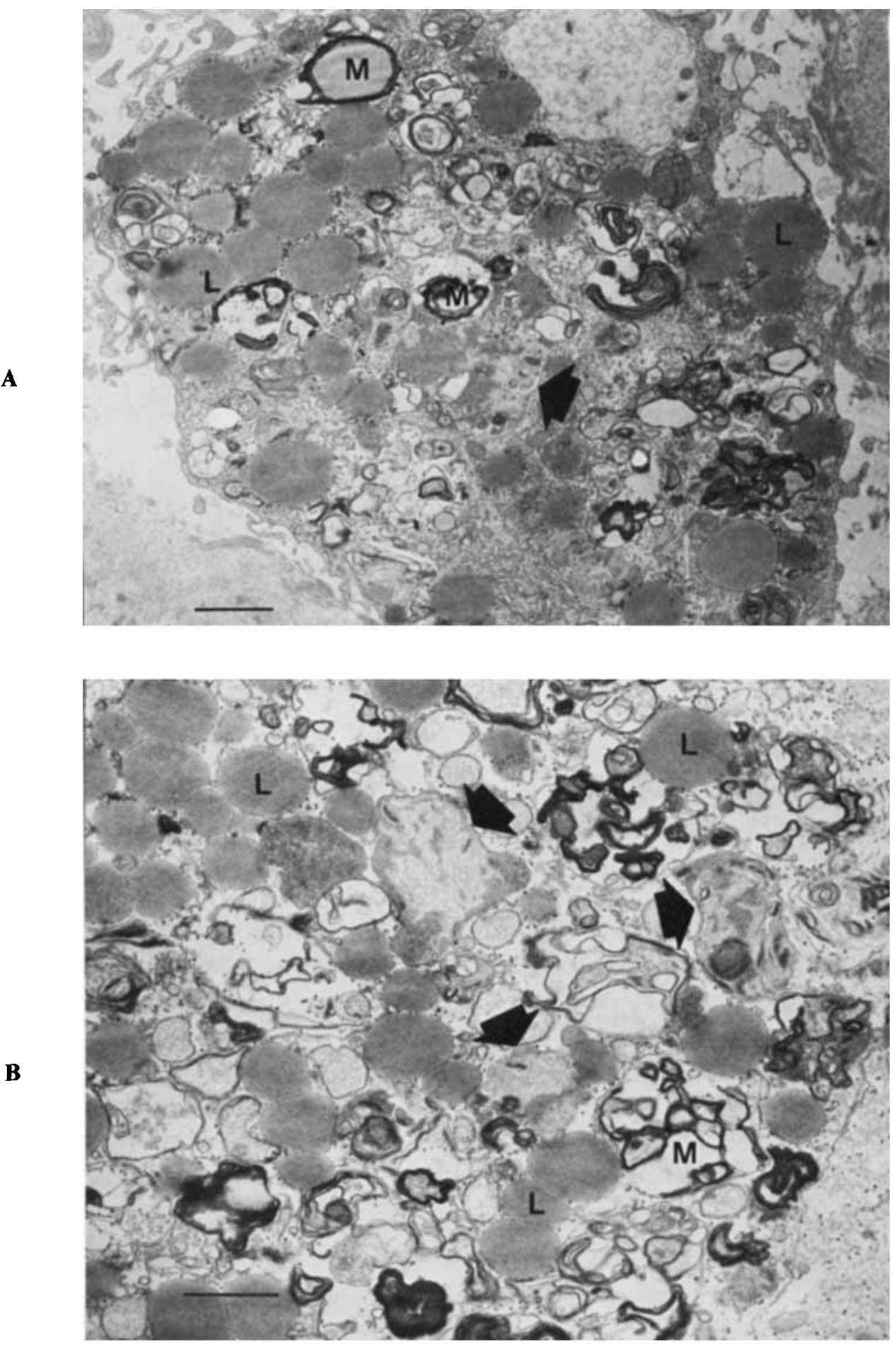

Figure 3A and B. Ultrastructure. Portions of macrophage cytoplasm. Lipidic droplets (L) and myelin figures (M) are numerous. Membranous inclusion bodies characteristic of Whipple's disease are designated with arrows. These inclusions contain many membrane profiles and degradation products of bacilli. The remnants of degenerated bacilli are shown cut in cross section in the inclusion in $\mathbf{A}$ and cut in longitudinal directions in the several inclusions marked in B. The magnification bar is $1 \mu \mathrm{m}$. 
stained with hematoxylin and eosin, and PAS with and without diastase.

By light microscopy there was a relatively acellular dense fibrous connective tissue capsule surrounding a central area of eosinophilic hyalin material containing focal aggregates of polymorphonuclear leukocytes and pockets of foamy macrophages (Figure 2A). These foamy macrophages contained PAS positive, diastase resistant curvilinear bodies (Figure 2B) which varied from weakly to markedly PAS positive, similar to those observed in the lamina propria of biopsies of the small bowel.

Ultrastructurally the hyalin material had a fibrillar matrix with pockets of cells. The cells were predominantly macrophages, but neutrophils and lymphocytes were also seen. The macrophages were laden with phagosomes representing numerous types of inclusions (Figures $3 \mathrm{~A}$ and B). Many phagosomes were lipidic; some were heterogeneous and resembled lipochrome inclusions. A few of the inclusions were best characterized as myelin figures. Also present were characteristic membranous phagosomes of the type seen in older patients with Whipple's disease. These phagosomes contained mostly membrane profiles; distinctive bodies representing degenerative bacilli (4) were present among the membranes. Extracellular intact bacilli or discrete bacilli laden phagosomes were not seen, but transitional forms with remnants of bacilli were present.

\section{DISCUSSION}

Synovial biopsy has been of value in the diagnosis and evaluation of therapeutic response in Whipple's disease (5). The present experience, belated in the course of our patient's disease, nevertheless indicates that biopsy of subcutaneous nodules in patients with poorly diagnosed arthritis may facilitate early recognition of Whipple's disease, an important consideration since effective treatment with antibiotics is at hand.

Acknowledgment. We would like to thank Dr. William O. Dobbins, III for review of the manuscript.

\section{REFERENCES}

1. LeVine ME, Dobbins WO, III: Joint changes in Whipple's disease. Semin Arthritis Rheum 3:79-93, 1973

2. Rodarte JR, Garrison CO, Holley KE, Fontana RS: Whipple's disease simulating sarcoidosis. Arch Intern Med 129:479-482, 1972

3. Carson FL, Martin JH, Lynn JA: Formalin fixation for electron microscopy; a re-evaluation. Am J Clin Pathol 59:865-873, 1973

4. Dobbins WO, III: Pathology of the intestine and colon, Diagnostic Electron Microscopy. Vol 1. Edited by BF Trump. New York, Wiley, 1978, pp 281- 283, 292-298

5. Hawkins CF, Farr M, Morris CJ, Hoare AM, Williamson $\mathrm{N}$ : Detection by electron microscope of rod-shaped organisms in synovial membrane from a patient with the arthritis of Whipple's disease. Ann Rheum Dis 35:502-509, 1976 